OPEN ACCESS

Edited by:

Sylvia Anton,

Institut National de la Recherche

Agronomique (INRA), France

Reviewed by:

Dan-Dan Zhang,

Lund University, Sweden

Sergio Angeli,

Free University of Bozen-Bolzano, Italy

Nicolas Durand,

University of Picardie Jules Verne,

France

*Correspondence:

Xinnian Zeng

zengxn@scau.edu.cn

Xincheng An

anxc@giabr.gd.cn

tThese authors have contributed equally to this work

Specialty section

This article was submitted to Invertebrate Physiology a section of the journal

Frontiers in Physiology

Received: 28 October 2019

Accepted: 29 May 2020

Published: 19 June 2020

Citation:

$X \cup Q$, Wu Z, Zeng X and An X (2020) Identification and Expression Profiling of Chemosensory Genes in Hermetia illucens via

a Transcriptomic Analysis.

Front. Physiol. 11:720.

doi: 10.3389/fphys.2020.00720

\section{Identification and Expression} Profiling of Chemosensory Genes in Hermetia illucens via a Transcriptomic Analysis

\author{
Qiyun $\mathrm{Xu}^{1,2 t}$, Zhongzhen $\mathrm{Wu}^{3 \dagger}$, Xinnian Zeng ${ }^{1 *}$ and Xincheng $\mathrm{An}^{2 *}$
}

1 Guangdong Engineering Research Center for Insect Behavior Regulation, College of Agriculture, South China Agricultural University, Guangzhou, China, ${ }^{2}$ Guangdong Key Laboratory of Animal Conservation and Resource Utilization, Guangdong Institute of Applied Biological Resources, Guangzhou, China, ${ }^{3}$ Guangzhou City Key Laboratory of Subtropical Fruit Trees Outbreak Control, Zhongkai University of Agriculture and Engineering, Guangzhou, China

The black soldier fly, Hermetia illucens, is a cosmopolitan insect of the family Stratiomyidae (Diptera). Chemosensory genes encode proteins involved directly in the detection of odorants. In this study, we sequenced the antennal transcriptome of $\mathrm{H}$. illucens adults to identify chemosensory genes. Putative unigenes encoding 27 odorant binding proteins (OBPs), five chemosensory proteins (CSPs), 70 odorant receptors (ORs), 25 ionotropic receptors (IRs), 10 gustatory receptors (GRs) and two sensory neuron membrane proteins (SNMPs) were identified. Tissue-specific expression profiles of the identified OBPs, CSPs and SNMPs were investigated using RT-PCR. Eight OBPs (HillOBP1-2, 9, 11-14, and 17), one CSP (HillCSP5) and one SNMP (HillSNMP1) were predominantly expressed in antennae. Further real-time quantitative PCR analyses revealed that the antennae-enriched unigenes also exhibited significant differences in expression between males and females. Among the sex-biased unigenes, six ORs showed female-biased expression, suggesting that these genes might participate in female-specific behaviors such as oviposition site searching. Sixteen ORs and two OBPs showed male-biased expression, indicating that they may play key roles in the detection of female sex pheromones. Our study is the first attempt to delineate the molecular basis of chemoreception in $\mathrm{H}$. illucens. Our data provide useful information for comparative studies on the differentiation and evolution of Dipteran chemosensory gene families.

Keywords: antennal transcriptome, chemosensory gene, identification, expression analysis, Hermetia illucens

\section{INTRODUCTION}

Olfaction plays a crucial role in insect behaviors, such as foraging, mating, oviposition and avoiding predators (Leal, 2013). The process of olfactory detection is mediated by a number of gene families including odorant-binding proteins (OBPs), chemosensory proteins (CSPs), sensory neuron membrane proteins (SNMPs), and olfactory receptors (ORs), ionotropic receptors (IRs) 
and gustatory receptors (GRs) (Clyne et al., 1999, 2000; Vosshall et al., 1999; Galindo and Smith, 2001; Benton et al., 2009; Vieira and Rozas, 2011). Each group of proteins participates in different steps of the chemosensory process. OBPs and CSPs are thought to bind, solubilize and transport hydrophobic odorants across the aqueous lymph surrounding the olfactory sensory neurons (OSNs) on the sensilla of antennae (Wojtasek and Leal, 1999; Sandler et al., 2000; Xu et al., 2005; Gomez-Diaz et al., 2013). ORs are responsible for the detection of odorants, whereas IRs are involved in sensing chemo-, thermo- and hygro-sensory stimuli (Ai et al., 2010, 2013; Grosjean et al., 2011; Silbering et al., 2011; Kain et al., 2013; Su and Carlson, 2013; Koh et al., 2014; Chen et al., 2015; Stewart et al., 2015; Gorter et al., 2016; Hussain et al., 2016; Knecht et al., 2016, 2017; Ni et al., 2016). GRs are thought to be involved in the detection of sugars, bitter tasting compounds and non-volatile pheromones (Clyne et al., 2000; Bray and Amrein, 2003; Dahanukar et al., 2007; Jiao et al., 2007; Sung et al., 2017) and carbon dioxide (Jones et al., 2007; Turner and Ray, 2009; Tauxe et al., 2013). SNMPs may play important roles in pheromone sensing based on their expression on the dendritic membrane of pheromone sensitive neurons (Benton et al., 2007; Gomez-Diaz et al., 2016).

After the comprehensive characterization of chemosensory genes in the two model species Drosophila melanogaster and Anopheles gambiae (Robertson et al., 2003; Rinker et al., 2013) a growing number of chemosensory genes have also been identified from many other Dipteran species based on sequence similarity. These Dipterans include Musca domestica (Scott et al., 2014) Bactrocera dorsalis (Wu et al., 2015; Jin et al., 2017), Calliphora stygia (Leitch et al., 2015), Glossina morsitans morsitans (Macharia et al., 2016), Mayetiola destructor Say (Andersson et al., 2014), Episyrphus balteatus and Eupeodes corollae (Wang et al., 2017), and Chlorops oryzae (Qiu et al., 2018).

The black soldier fly, Hermetia illucens, is a cosmopolitan Dipteran. The larvae of $H$. illucens play a pivotal role in terms of both environmental and economic aspects for waste disposal and processing. H. illucens larvae are useful in manure management for controlling housefly populations and converting organic waste into useful products such as compost (Newton et al., 2005). Like other many Dipteran species, $H$. illucens adults have a sensitive olfactory system and use a wide range of environmental chemical cues to locate food, mates, and egg-laying sites (Booth and Sheppard, 1984; Tomberlin and Sheppard, 2001, 2002). $H$. illucens could potentially be used to decompose landfills if adult flies can be guided to deposit their eggs there. A better understanding on the molecular components of the H. illucens olfactory system is an initial step toward this type of practical application. Identification and characterization of chemosensory genes are also important steps toward understanding their evolution and primary functions. The objective of this study is to identify candidate chemosensory genes encoding OBPs, CSPs, ORs, IRs, GRs, and SNMPs by generating and analyzing the antennal transcriptome of $H$. illucens adults. Genetic and phylogenetic analyses as well as expression profiling of identified chemosensory genes were also carried out to gain insights on their potential functions.

\section{MATERIALS AND METHODS}

\section{Insects, Tissues Collection and RNA Isolation}

Hermetia illucens adults were obtained from a colony maintained year-round in the laboratory of the Guangdong Public Laboratory of Wild Animal Conservation and Utilization, Guangdong Institute of Applied Biological Resources in Guangzhou City, Guangdong Province, China. The colony has been maintained at $28^{\circ} \mathrm{C}$ with a photoperiod of $14: 10 \mathrm{~h}$ (Light: Dark) and $70 \%$ relative humidity.

For transcriptomic analyses, 150 pairs of antennae were collected separately from both females and males of $H$. illucens adults. For RT-PCR analysis, 50 tissues of antennae, mouthparts, foreleg tarsus, wings and genitals were separately obtained from adult males and females. Three replicates were generated for each tissue set.

Total RNA was isolated from homogenized tissues using Trizol reagent (Invitrogen, Carlsbad, CA, United States) following the manufacturer's instructions and then treated with RNase-free DNase I (TaKaRa, Dalian, China) to remove potential genomic DNA contamination. RNA integrity was monitored on $1 \%$ agarose gel, and assessed with an Agilent 2100 Bioanalyzer (Agilent Technologies, Santa Clara, CA, United States). RNA concentration and purity were analyzed on a NanoDrop ND2000 Spectrophotometer (Nanodrop Technologies, Wilmington, DE, United States).

\section{cDNA Library Construction, Sequencing and de novo Assembly}

cDNA libraries were constructed with $1.5 \mu \mathrm{g}$ purified RNA using a TruSeq RNA Sample Preparation Kit (Illumina, San Diego, CA, United States) following the manufacturer's instruction. Library preparations were sequenced using the Illumina HiseqTM 2500 platform (San Diego, CA, United States) and paired-end reads were generated. After sequencing, raw reads were firstly processed through in-house perl scripts. Clean reads were obtained from raw data after removing reads containing adapter, unknown (poly- $\mathrm{N}$ ) and low-quality reads. Clean reads assembly was accomplished using Trinity (Version: r2013-11-10) with the default parameters after combining the male and female clean reads (Grabherr et al., 2011). The largest assembly sequences were deemed to be unigenes. The clean reads from the antennal transcriptome of $H$. illucens were uploaded to the NCBI Sequence Read Archive (SAMN12915779).

\section{Functional Annotation}

BLASTx searches were carried out against sequences in the NCBI non-redundant protein (nr) protein database with a cutof $E$-value of $10^{-5}$. Unigenes were also annotated using other databases including NCBI non-redundant nucleotide (nt), SwissProt, the Kyoto Encyclopedia of Genes and Genomes (KEGG), Gene Orthology (GO) and Cluster of Orthologous Groups of proteins (COG). 


\section{Gene Identification}

To identify chemosensory genes from $H$. illucens, known OBPs, CSPs, ORs, IRs, GRs, and SNMPs from other Dipteran insects were selected as queries to search the $H$. illucens antennal transcriptome. Query OBPs were from D. melanogaster, $B$. dorsalis, Ceratitis capitata, and M. domestica. Query CSPs were from D. melanogaster, B. dorsalis, $M$. domestica and G. morsitans. Query ORs were from D. melanogaster, B. dorsalis, C. stygia, and $M$. domestica. Query GRs were from $D$. melanogaster, C. stygia and C. capitata for IRs; D. melanogaster, B. dorsalis and C. stygia. Query SNMPs were from D. melanogaster, B. dorsalis, and M. domestica (Supplementary Table S1). tBLASTn was used to identify candidate unigenes encoding OBPs, CSPs, ORs, IRs, GRs, and SNMPs against the H. illucens antennal transcriptome with a cut-of $E$-value of $10^{-5}$. Identified candidate unigenes were manually checked using BLASTx against the NCBI non-redundant protein sequences database. Potential open reading frames (ORFs) of candidate chemosensory genes were predicted using the NCBI ORF Finder ${ }^{1}$. Alignments of amino acid sequences were performed using MAFFT (Version: 7.308) (E-INS-I parameter set) (Katoh and Standley, 2013) and visualized with Geneious (Version: 9.1.3) (Kearse et al., 2012). Protein domains (including transmembrane domains and signal peptides) were predicted using the InterProScan tool plug-in in Geneious (Quevillon et al., 2005). tBLASTn searches were used to determine the scaffold location, position and intronexon organization of the candidate chemosensory genes in each H. illucens's genomic scaffold (GCA_009835165.1). Candidate unigenes coding for chemosensory genes and their corresponding reference genes were listed in Supplementary Table S2.

\section{Phylogenetic Analysis}

Amino acid sequences of candidate OBPs, CSPs, ORs, IRs, GRs, and SNMPs from H. illucens were aligned together with proteins from other Dipterans. The sequences from other Dipterans used for building phylogenetic trees are listed in Supplementary Table S3. Sequence alignments were generated using Clustal Omega (Sievers et al., 2011), and maximum-likelihood trees were constructed using FastTree2 (Jones-Taylor-Thornton amino acid substitution model) with default settings and 1000 bootstrap replicates (Price et al., 2010). Phylogenetic trees were colored and arranged using FigTree (Version: 1.4.2).

\section{Analyses of Expression Levels Based on FPKM}

Clean reads were mapped back onto the assembled unigenes and read count for each unigene was obtained from the mapping results. The expression levels of these unigenes were calculated as the fragments per kilobase per million mapped fragments (FPKM) method (Trapnell et al., 2010).

\section{RT-PCR and Real-Time Quantitative PCR}

The expression profiles of 27 OBPs, 5 CSPs, and 2 SNMPs among various tissues (antennae, mouthparts, foreleg tarsus,

\footnotetext{
${ }^{1}$ https://www.ncbi.nlm.nih.gov/orffinder/
}

wings, and genitals) were initially evaluated using RT-PCR. Total RNA was isolated from these tissues, and cDNA was synthesized using a PrimeScript RT reagent Kit (Takara). In our pre-experiment, $\alpha$-tubulin $(\alpha-T U B)$ and glyceraldehyde-3phosphate dehydrogenase (GAPDH) were evaluated as the most stable reference genes for gene expression profiling across the different tissues (male antennae, female antennae mouthparts, foreleg tarsus, wings, and genitals) using Normfinder (Andersen et al., 2004) and BestKeeper (Pfaffl et al., 2004) (Supplementary Table S4). Therefore, $\alpha-T U B$ and GAPDH genes were used as controls to assess the cDNA integrity. PCR reactions were conducted using a Bio-Rad thermal cycler (Bio-Rad, Hercules, CA, United States) with the same cycling parameters as our previous study (Cui et al., 2019). PCR products were analyzed by electrophoresis in $1.5 \%$ agarose gels. Each PCR reaction was repeated twice with independently isolated RNA samples.

Based on the RT-PCR results, unigenes encoding OBPs, CSPs, IRs, and ORs that were predominantly or exclusively expressed in antennae were analyzed again for more accurate estimation using real-time quantitative PCR (RT-qPCR). RT-qPCR analysis was conducted using a LightCycler 480 system (Roche Applied Science, Basel, Switzerland). PCR reaction conditions were the same to those used in our previous study (Cui et al., 2019). Each RT-qPCR reaction was performed in three technical replications with three independent biological replications, and PCRs with no template (nuclease-free water) were used as negative controls. RT-qPCR analysis was performed using the LightCycler 480 gene scanning software. Relative gene expression level was quantified using the comparative $2^{-\Delta \Delta C T}$ method (Livak and Schmittgen, 2001 ) and calculated relative to $\alpha-T U B$ and GAPDH. All genespecific primers were designed using Primer3 (Version: 4.1.0) (Supplementary Table S5).

\section{Statistical Analysis}

Data analysis was conducted using SPSS 22.0 (SPSS Inc., Chicago, IL, United States). The significant difference analysis of each gene among various tissues was determined using a one-way nested analysis of variance (ANOVA), following by Duncan's new multiple range test $(\alpha=0.05)$. Values are presented as mean $\pm \mathrm{SE}$. The GraphPad Prism 6.0 software (GraphPad Inc, San Diego) was used to perform the figures.

\section{RESULTS}

\section{Transcriptome Sequencing and de novo Assembly}

In total, 118.56 million raw reads (17.78 GB raw data) were obtained from the antennal transcriptome of $H$. illucens. Additionally, 117.99 million of clean reads (17.70 GB clean data) were generated after filtering adapters and low-quality raw sequences. The clean reads were assembled into 70,124 unigenes with an N50 of 2,102 bp, average length of 1,002 bp (Supplementary Table S6). Length distribution analysis

${ }^{2}$ http://primer3.ut.ee/ 
indicated 33,523 unigenes, which accounted for $47.8 \%$ of all unigenes, were longer than 500 bp (Supplementary Table S7).

\section{Functional Annotation}

There were 24,837 (35.42\%), 11,836 (16.88\%), 16,633 (23.72\%), 7,051 (10.06\%), 7,873 (11.23\%), and 17,675 (25.21\%) unigenes that had homologous sequences in NCBI-nr, NCBI-nt, SwissProt, GO, COG and KEGG databases, respectively. In total, $26,259(37.45 \%)$ unigenes were annotated and the remaining unigenes were unmappable at present based on the sequence homology (Supplementary Figure S1A). Low homology with other insect species in NCBI was observed. The highest match percentage $(6.84 \%)$ was identified with sequences of Lucilia cuprina followed by sequences of $M$. domestica (5.68\%) and C. capitata (5.65\%) (Supplementary Figure S1B). Gene ontology (GO) analysis was used to classify unigenes into different functional categories (Supplementary Figure S1C). In the 'biological process' category, the subcategories "cellular," "metabolic" and "single-organism" process were the most represented. In the 'cellular component' category, the subcategories "cell" and "cell part" and "membrane" were the most represented. In the 'molecular function' category, the subcategories "binding" and "catalytic activity" were most represented.

\section{Transcript Abundance in $\boldsymbol{H}$. illucens Antennae}

The expression levels of all unigenes are given in Supplementary Table S8. Unigenes with FPKM values $\geq 1,000$ were defined as highly expressed, those with FPKM 200 1,000 were defined as moderately expressed, and those with FPKM $\leq 200$ were defined as weakly expressed. According to these criteria, two unigenes coding for CSPs (named HillCSP1 and 2) and three unigenes coding for OBPs (named HillOBP1, 2, and 3) were abundant in antennae with 24,339, 2,654, 5,664, 5,368, and 4,918 FPKM, respectively, suggesting their potential roles in odorant detection. Other highly abundant unigenes included those coding for the cytochrome oxidase subunit 2 (mitochondrion) (CL10816.Contig1_All, 4,103 FPKM), the cytochrome oxidase subunit 1 (mitochondrion) (CL14318.Contig1_All, 2,942 FPKM), and cytochrome oxidase subunit 3 (mitochondrion) (Unigene12013_All, 2,908 FPKM). Interestingly, a unigene encoding a takeout-like protein was also highly expressed in H. illucens antennae (Unigene14734_All, 2,446 FPKM).

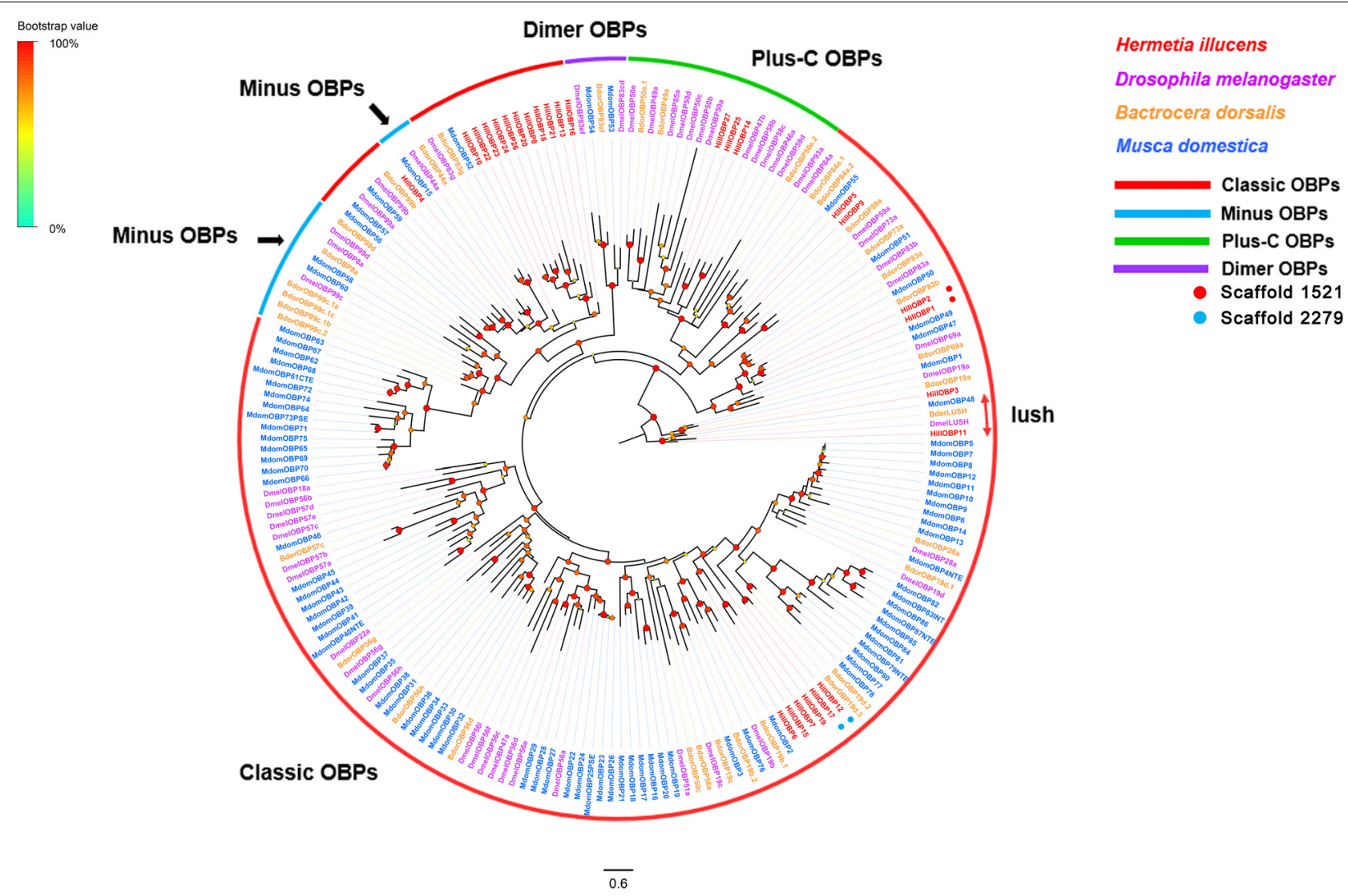

FIGURE 1 | Maximum likelihood tree of candidate OBPs from H. illucens and other Dipteran insects. The distance tree was rooted by the lush orthologs. Branch support was estimated using 1000 bootstrap replicates, and bootstrap values were displayed with color circles at the branch nodes. Bars indicate the expected number of amino acid substitutions per site. Classic OBPs are in red; Minus OBPs in blue; Plus-C OBPs in green; Dimer OBPs in purple. OBPs located in the scaffold 1521 are highlighted with red dots. OBPs located in the scaffold 2279 are highlighted with blue dots. 


\section{Candidate Odorant Binding Proteins (OBPs)}

Twenty-seven OBP-encoding unigenes (HillOBP1-27) were identified from the $H$. illucens antennal transcriptome (Supplementary Table S2: Sheet 1). The $27 \mathrm{H}$. illucens OBPs were distributed among 16 different scaffolds, with HillOBP8/20/21/22/23/24/26 collocated in scaffold 2649, HillOBP4/10/16 collocated in scaffold 1747, HillOBP1/2 located in scaffold 1521, HillOBP3/15 located in scaffold 2041, HillOBP12/17 located in scaffold 2279. All identified OBP unigenes except three (HillOBP5, 21 and 22) had a full-length ORFs encoding proteins with 130 to 233 amino acid residues. All these predicted proteins have a putative signal peptide at the $\mathrm{N}$ terminal region. Sequence identities of predicted OBPs with those from other Dipterans in the NCBI-nr database ranged from 26.55 to $76.55 \%$, with an average of $39.48 \%$. According to the number and location of the conserved cysteines (Hekmat-Scafe et al., 2002), twenty four OBPs (HillOBP1-4, $6-20,23-27)$ were classified as classic OBPs, with the typical six conserved C-residues (Supplementary Figure S2), and three OBPs (HillOBP14, 25, and 27) were classified as plus-C OBPs, with four to six additional cysteine residues in addition to the six conserved cysteine residues. Phylogenetic analyses of OBPs from $H$. illucens and other three Dipteran species (D. melanogaster, B. dorsalis, and M. domestica) showed that all the $H$. illucens OBPs formed distinct clades based on an insect OBP classification system, and segregated into the classic OBP and plus-C OBP sub-families (Figure 1). FPKM value analysis revealed that five OBPs (HillOBP1-5) were highly expressed in $H$. illucens antennae (FPKM >1,000) (Supplementary Table S2: Sheet 1).

\section{Candidate Chemosensory Proteins (CSPs)}

Five CSP-encoding unigenes (HillCSP1-5) were identified (Supplementary Table S2: Sheet 2). The five $H$. illucens CSPs were distributed among two different scaffolds, with HillCSP1/2/4/5 collocated in scaffold 1059, HillCSP3 located in scaffold 1518. All the unigenes encoding CSPs have full-length ORFs encoding proteins with 110-128 amino acid residues, including four highly conserved cysteine residues and a signal peptide (Supplementary Figure S3). A phylogenetic tree was constructed using all predicted HillCSPs together with those from other Dipteran species. The CSPs of $H$. illucens formed four distinct clades marked as A, B, $\mathrm{C}$, and D in Figure 2. Based on FPKM values, HillCSP1 is

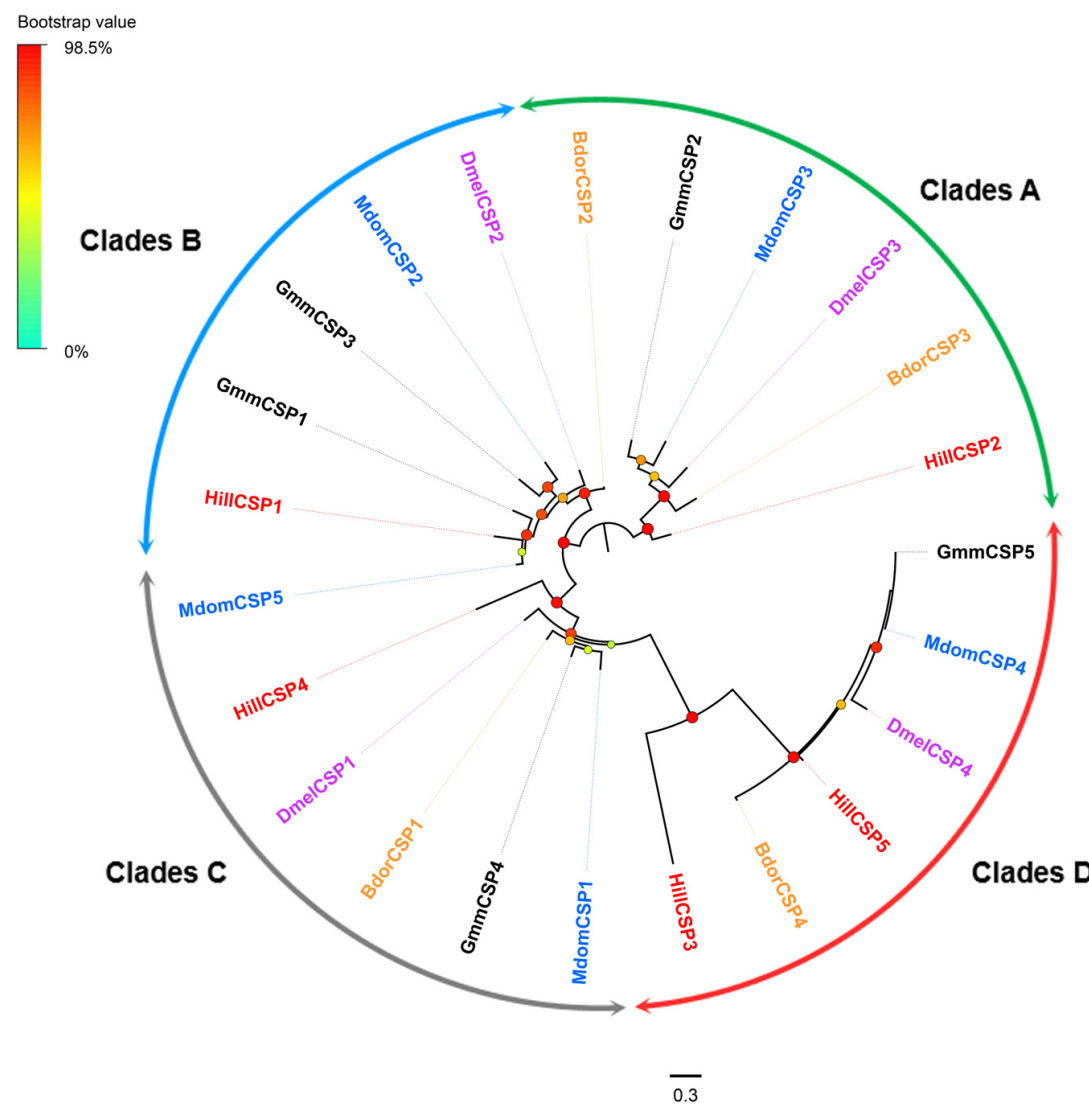

Hermetia illucens

Drosophila melanogaster

Bactrocera dorsalis

Musca domestica

Glossina morsitans morsitans

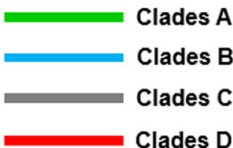

FIGURE 2 | Maximum likelihood tree of candidate CSPs from H. illucens and other Dipteran insects. Branch support was estimated using 1000 bootstrap replicates, and bootstrap values were displayed with color circles at the branch nodes. The scale bar indicate the expected number of amino acid substitutions per site. Clades A are in green; Clades B in blue; Clades $C$ in gray; Clades D in red. 
the most highly expressed in antennae with FPKM $>24,000$, followed by HillCSP2 (FPKM >2,000) (Supplementary Table S2: Sheet 2).

\section{Candidate Odorant Receptors (ORs)}

Seventy OR-encoding unigenes were identified, including one Orco (HillOR1) and 69 conventional OR genes (HillOR270) (Supplementary Table S2: Sheet 3). The $70 \mathrm{H}$. illucens ORs were distributed among 38 different scaffolds, with HillOR62/69 located in scaffold 1002, HillOR6/38/63 collocated in scaffold 1069, HillOR10/18/20/30 collocated in scaffold 1106, HillOR32/44/53 collocated in scaffold 1295, HillOR2/3/7/14/23/40/47/55/60 collocated in scaffold 1361, HillOR51/57 collocated in scaffold 1950, HillOR24/29/33/41/49 collocated in scaffold 2127, HillOR15/54 collocated in scaffold 2245, HillOR5/25/58 collocated in scaffold 2284, HillOR13/19/26/31/43 collocated in scaffold 2791, HillOR37/61 located in scaffold 281, HillOR21/50 located in scaffold 300,
HillOR39/64 located in scaffold 440, HillOR35/52 located in scaffold 793, HillOR22/59 located in scaffold 914. Among these OR unigenes, 48 have full-length ORFs encoding proteins with 360 to 477 amino acid residues. Four of the predicted proteins have 4-8 transmembrane domains (TMDs). The highly conserved co-receptor HillOR1 shared $82.53 \%$ identity with a co-receptor from C. capitata (XP_012156143), while other HillORs shared $21.51-82.53 \%$ identity with those from other Dipterans. A phylogenetic tree was constructed using our identified ORs along with a data set containing representative ORs from three other Dipterans, including D. melanogaster, $C$. stygia and $M$. domestica (Figure 3). The vast majority of HillORs formed several species-specific clades, and no ORs in $H$. illucens clustered with orthologs from other species. Among these HillORs, HillOrco had the highest expressional level $(\mathrm{FPKM}=1,159.56)$, whereas the other HillORs are weakly expressed (FPKM: 1.12 140.25) (Supplementary Table S2: Sheet 3).
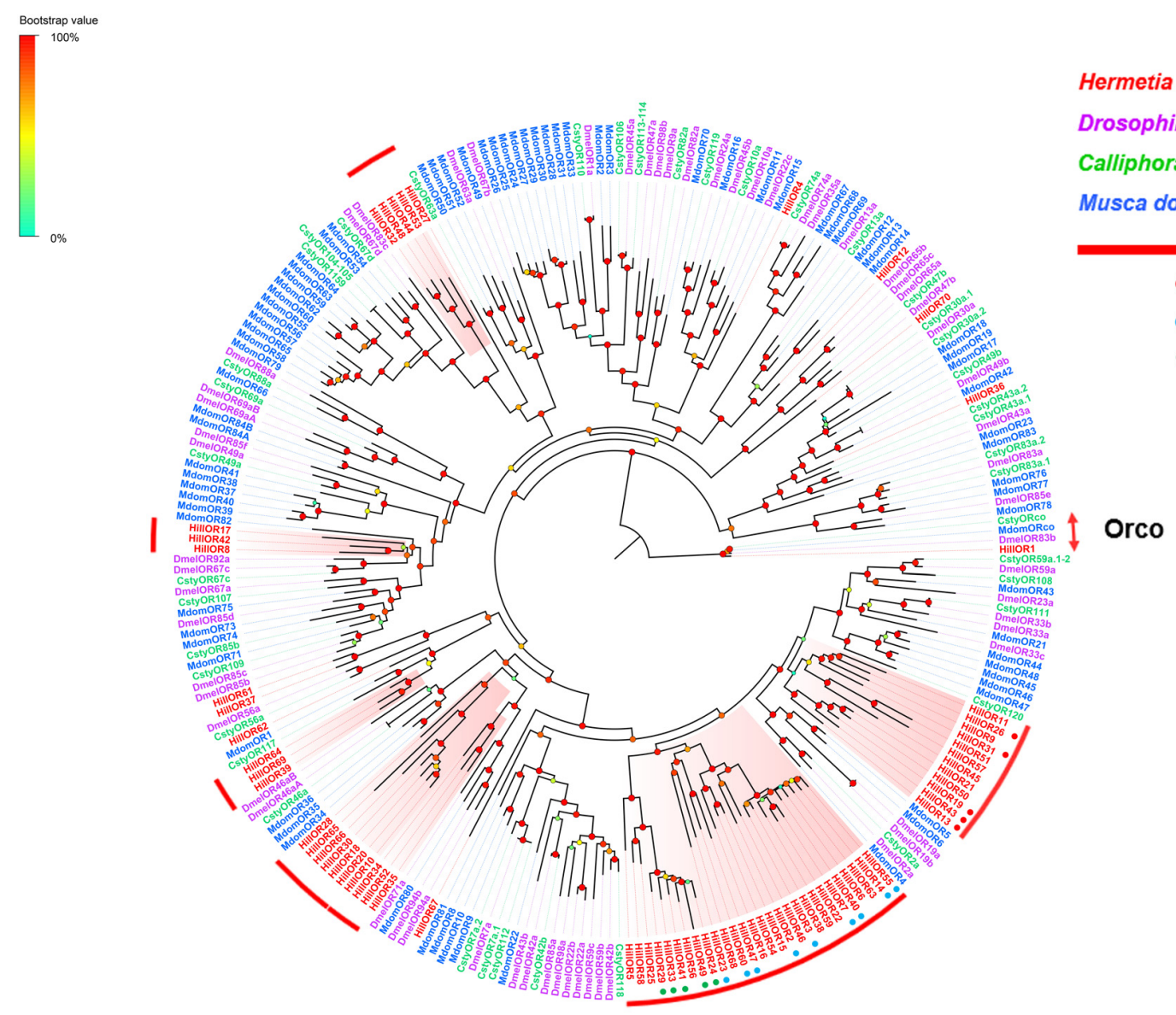

FIGURE 3 | Maximum likelihood tree of candidate ORs from $H$. illucens and other Dipteran insects. The distance tree was rooted by the conservative ORco orthologs. The species-specific clades are labeled with red. Branch support was estimated using 1000 bootstrap replicates, and bootstrap values were displayed with color circles at the branch nodes. The scale bar indicate the expected number of amino acid substitutions per site. ORs located in the scaffold 2791 are highlighted with red dots. ORs located in the scaffold 1361 are highlighted with blue dots. ORs located in the scaffold 2171 are highlighted with green dots. 


\section{Candidate lonotropic Receptors (iGluRs/IRs)}

Twenty-five iGluR/IR-encoding unigenes were identified (Supplementary Table S2: Sheet 4). Of these iGluR/IR unigenes, twenty have full-length ORFs encoding proteins with at least 426 amino acid residues. The $25 \mathrm{H}$. illucens iGluR/IR were distributed among 21 different scaffolds, with HillCG5621.1/ CG5621.2/IR93a collocated in scaffold 2289, HilliR75c.1/75c.2 located in scaffold 435 and HillIR40a.1/40a.2 located in scaffold 2311. Distinct clades were observed in a phylogenetic tree constructed with our identified iGluRs/IRs and orthologs from D. melanogaster and $M$. domestica (Figure 4). Among the identified iGluR/IRs, 17 antennal IRs clustered with previously reported "antennal" orthologs HilliR8a, 25a, 21a, 40a.1, 40a.2, $41 a, 64 a, 75 c .1,75 c .2,75 d .1,75 d .2,75 d .3,76 b, 84 a, 92 a$, and $93 a$; and were clearly separated from those non-NMDA iGluRs, NMDA iGluRs and divergent IRs clades. Interestingly, a usually conserved "antennal" ortholog, IR76a, was absent from $H$. illucens. Instead, two IR75c orthologs (IR75c.1 and
75 c.2), and three IR75d orthologs (IR75d.1, 75d.2, and 75d.3) was found in $H$. illucens. FPKM value analysis indicated that all these iGluR/IR unigenes were expressed at very low levels (the average FPKM value of 7.55), and only three "IR co-receptor" orthologs (HillIR25a, 8a, and 76b) were expressed at relatively higher levels (FPKM > 20).

\section{Candidate Gustatory Receptors (GRs)}

Ten GR-encoding unigenes were identified, and four of them encode full-length proteins with 6-8 TMDs (Supplementary Table S2: Sheet 5). The $10 \mathrm{H}$. illucens GRs were distributed among 10 different scaffolds. Potential functions of GRs identified from $H$. illucens could be inferred from their phylogenetic relationship with GRs previously well characterized from other Dipteran species (Figure 5). HillGR1, 2, and 3 were clustered with carbon dioxide GRs (DmelGR21a and 63a) (Jones et al., 2007; Kwon et al., 2007). HillGR5 was clustered with the Drosophila saponin receptor DmelGR28b (Sang et al., 2019). HillGR10 were clustered with Drosophila sugar receptors (DmelGR64a)

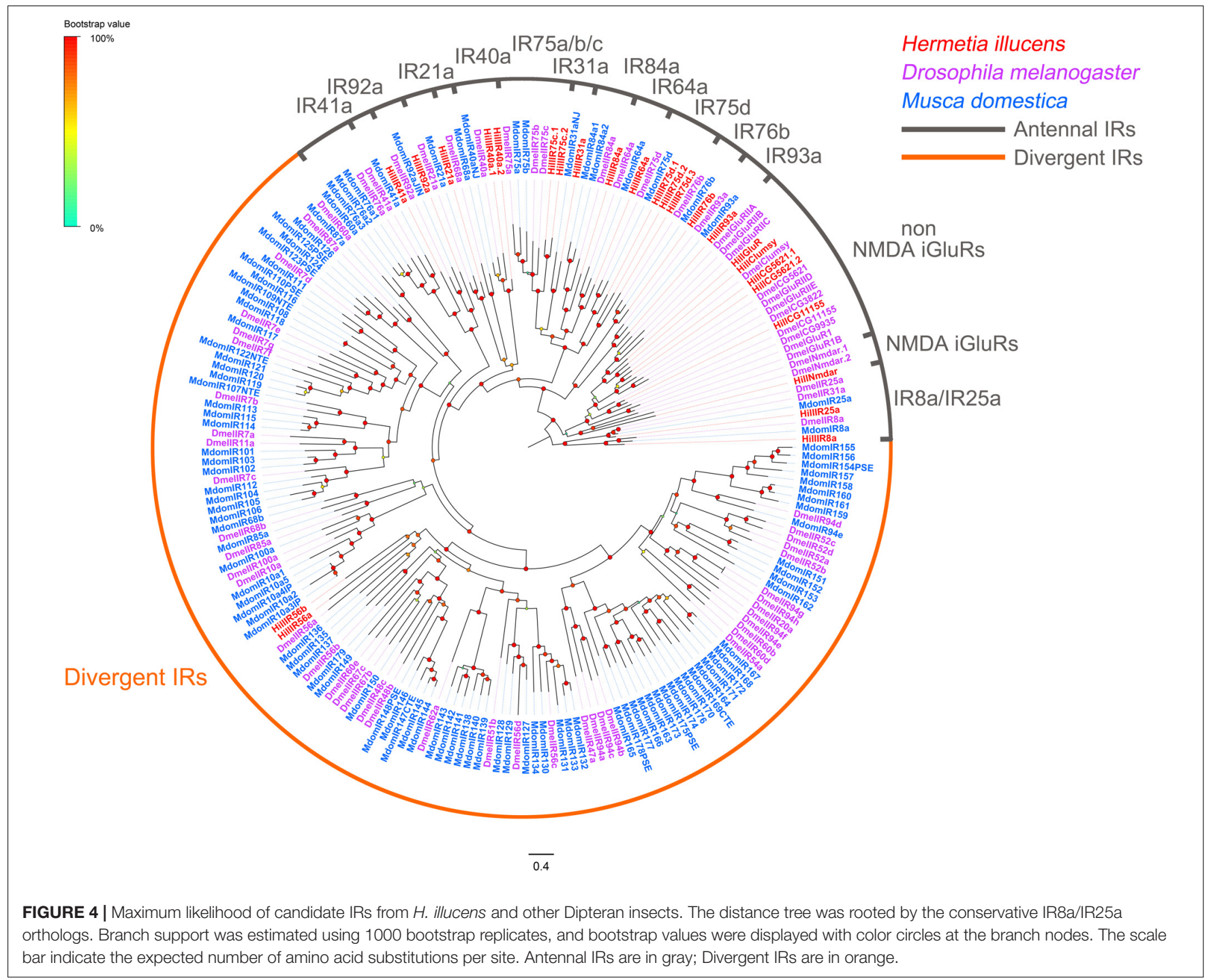




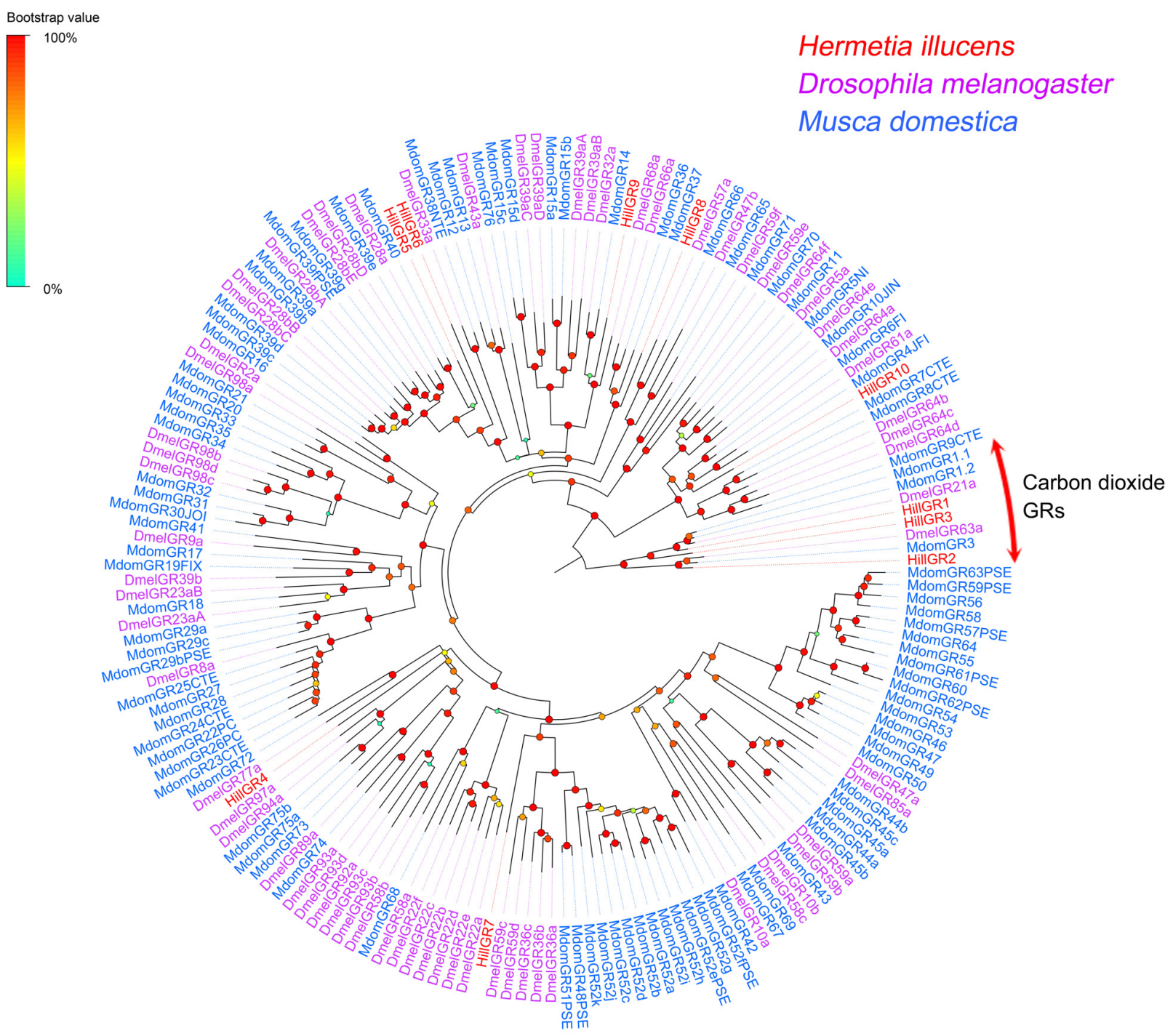

0.5

FIGURE 5 | Maximum likelihood of candidate GRs from $H$. illucens and other Dipteran insects. The distance tree was rooted by the conservative carbon dioxide GRs orthologs. Branch support was estimated using 1000 bootstrap replicates, and bootstrap values were displayed with color circles at the branch nodes. The scale bar indicate the expected number of amino acid substitutions per site.

(Dahanukar et al., 2007). All putative GR-encoding genes were expressed at very low levels, with an average FPKM value of 5.87, except three genes encoding carbon dioxide GRs, which showed higher expression levels with FPKM values 24.49, 10.22, and 9.3 , respectively.

\section{Candidate Sensory Neuron Membrane Proteins (SNMPs)}

Two SNMP-encoding unigenes were identified. These two unigenes have full-length ORFs encoding proteins with two TMDs (Supplementary Table S2: Sheet 6). The two H. illucens SNMPs were distributed among two different scaffolds. Based on a phylogenetic analysis, all SNMPs were classified into two distinct subfamilies, SNMP1 and SNMP2 (Supplementary Figure S4). HillSNMP1 was clustered with the SNMP1 subfamily, while HillSNMP2 clustered with the SNMP2 subfamily. One SNMP unigene, HillSNMP1, was expressed at relatively high levels with an FPKM value of 1,338.07 in H. illucens antennae (Supplementary Table S2: Sheet 6).

\section{Tissue- and Sex-Specific Expression}

RT-PCR showed that eight OBP-encoding unigenes (HillOBP12, 9, 11-14, and 17) were almost exclusively expressed in antennae, while HillOBP3 and 15 were highly expressed in 


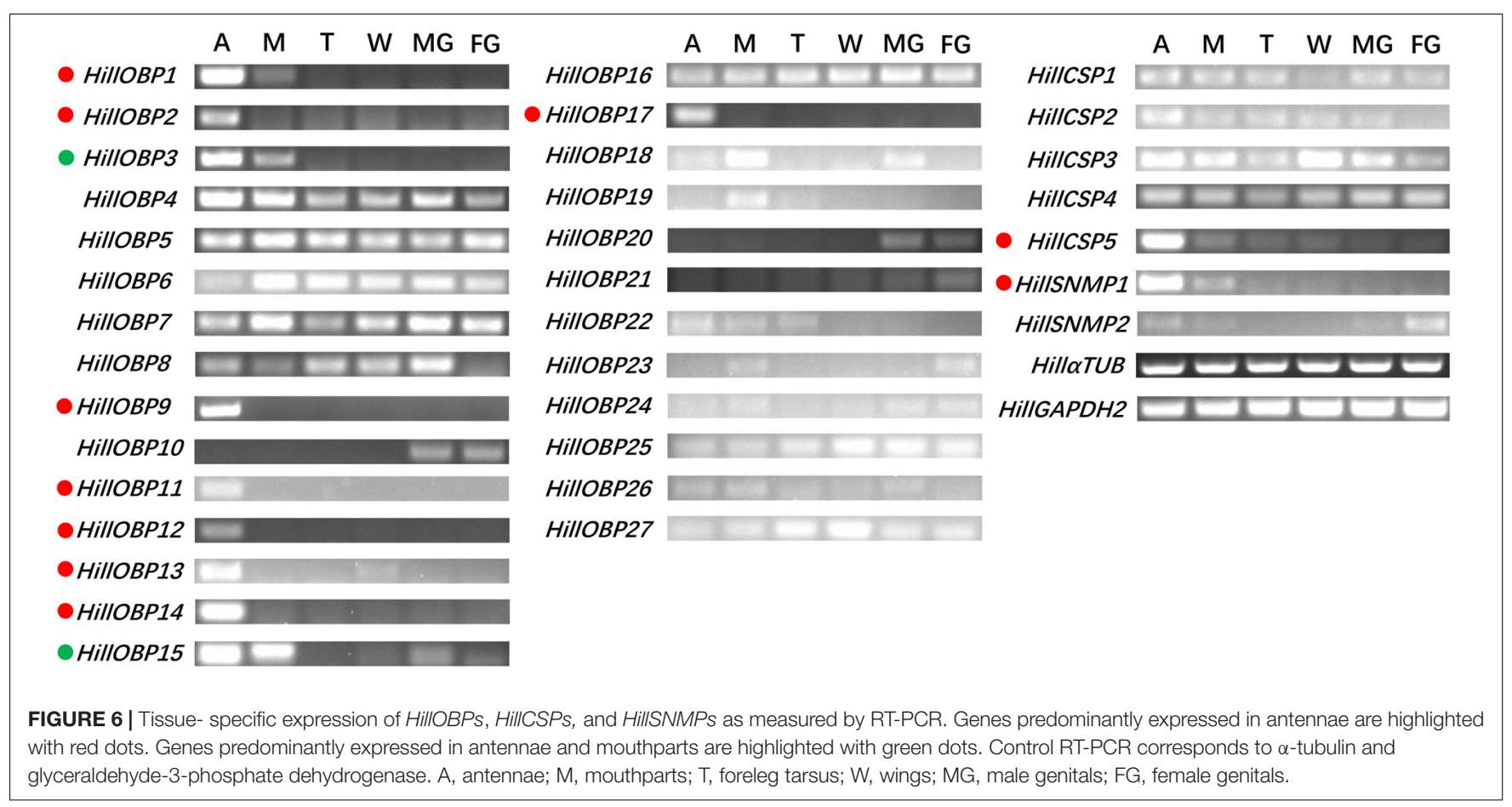

both antennae and mouthparts (Figure 6). The remaining OBPencoding unigenes were abundant in multiple tissues. Among the CSP-encoding unigenes, HillCSP5 was exclusively expressed in antennae, while other HillCSPs were present in multiple tissues. In addition, HillSNMP1 were mainly expressed in antennae.

In the OR-encoding unigenes, there were 15 ORs (HillOR2$6,9,10,14,15,19,39,40,52,59$, and 63) and six (HillOR32, $44,56,57,60$, and 66 ) with significantly higher expression in the male and female antennae, respectively (Figure 7A). Among antennal IR-encoding unigenes, all were equally expressed in the male and female antennae (Figure 7B). Among OBPencoding unigenes, there were two (HillOBP1 and 2) with significantly higher expression in males. In addition, HillCSP5 and HillSNMP1 were equally expressed in the antennae of both sexes (Figure 7C).

\section{DISCUSSION}

Hermetia illucens, a representative species of the Stratiomyidae family, is evolutionally different from many well characterized Dipteran species such as $D$. melanogaster, $M$. domestica, B. dorsalis, C. stygia, G. morsitans, and M. destructor (Brammer and von Dohlen, 2007). Homology analyses of the identified $H$. illucens chemosensory genes along with orthologs from other Dipterans show clear separation of the H. illucens genes from the ones from other flies (Supplementary Figure S1B). However, $H$. illucens adults exhibit both similarity and differences in mating and oviposition behaviors with $D$. melanogaster and other flies. For example, $H$. illucens males exhibit territorial lekking behavior during mating (Tomberlin and Sheppard, 2001) and can also detect odors released due to the action of bacteria for oviposition (Zheng et al., 2013). Thus, systematic research on chemoreception may provide valuable information on understanding the evolution of the insect olfactory system. H. illucens larvae play pivotal roles in both environmental and economic aspects of waste disposal and processing. Artificial release of $H$. illucens adults is a well-known method for waste treatment, but it is difficult to establish populations in the field. Understanding of the molecular recognition mechanisms and the molecular basis for egg-deposition behavior in response to environments has important implications for population establishment after release. With the recent release of the $H$. illucens genome, chemosensory genes have been annotated (Zhan et al., 2020). In this study, all the chemosensory genes identified in the antennal transcriptome of $H$. illucens are present in the genome. This result supports strongly the quality of the transcriptomic assembly.

OBPs identified in $H$. illucens are not very conserved in comparison with those from other Dipteran species. Phylogenetic analysis reveals that only a small subset of OBPs (such as HillOBP1, 2, 3, 5, 9, 11, and 14) identified in H. illucens have homologs from other Dipterans (Figure 1). Only two types of OBPs are found in $H$. illucens: classic and plusC. Minus and dimer OBPs which have been reported from other Dipteran species were not found in $H$. illucens. The missing of Minus and dimer OBPs may reflect physiological and evolutionary differences between $H$. illucens and other Dipteran species. Nevertheless, there are some OBPs that are conserved and have orthology relationships with counterparts from other Dipterans. For example, HillOBP1 and 2 are homolog to $O B P 83 b$ found in B. dorsalis, HillOBP11 is a homolog to Lush found in D. melanogaster, and HillOBP9 is a homolog to 


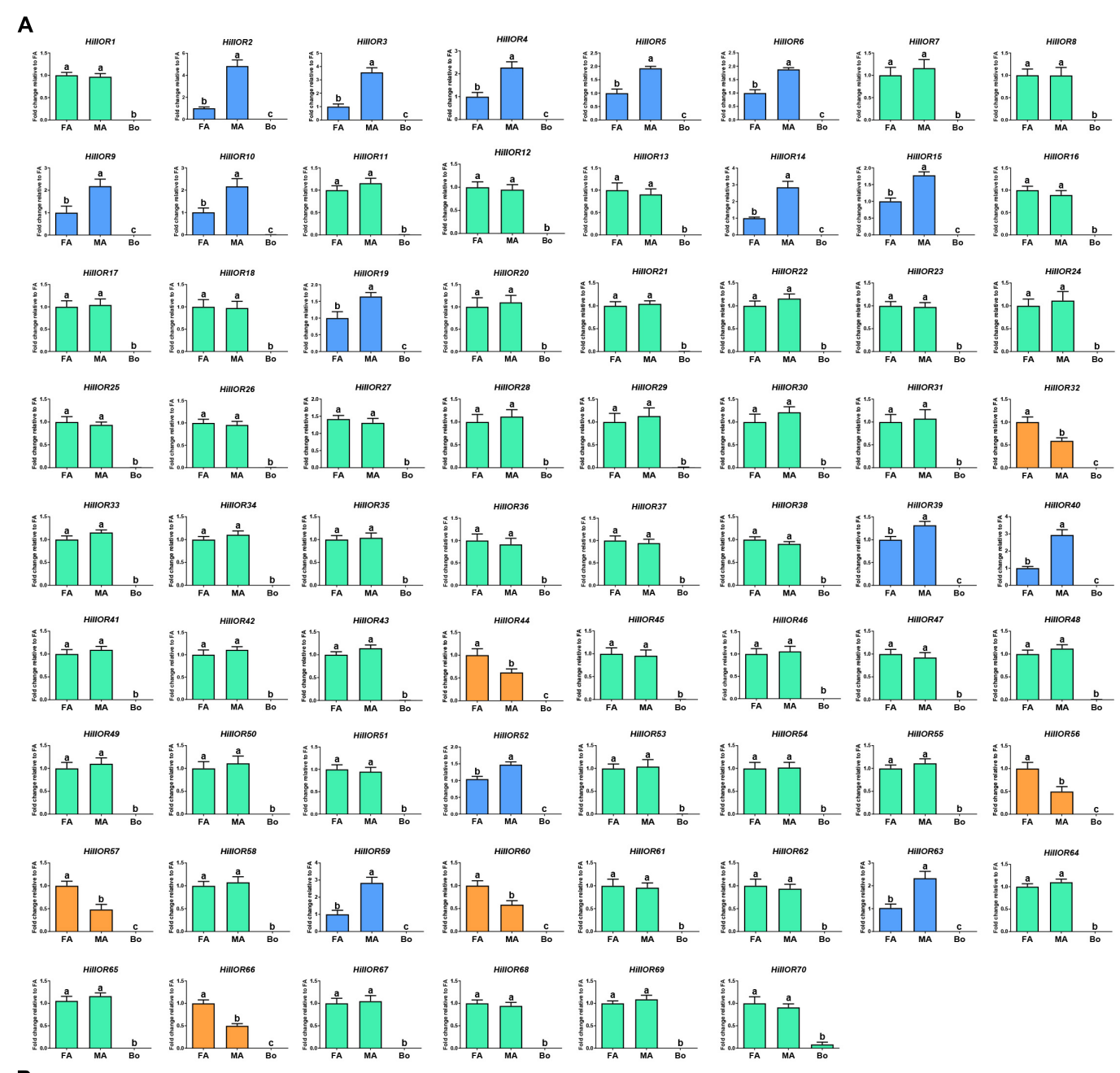

B

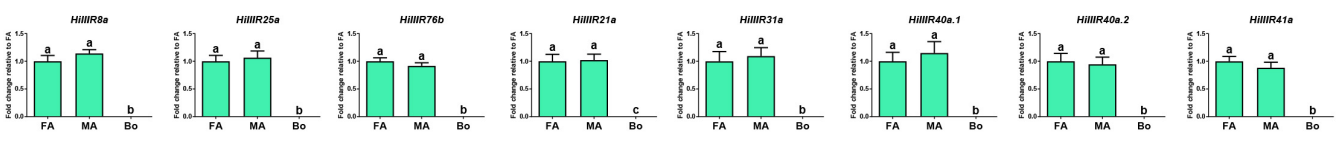

$\underset{\text { FA }}{20}$

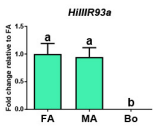

C
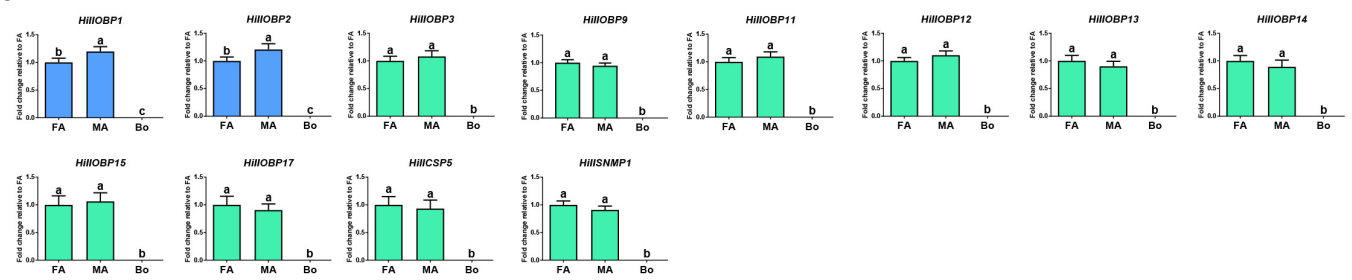

FIGURE 7 | Transcript levels of the olfactory-related genes in different tissues as measured by RT-qPCR. (A) ORs. (B) Antennal IRs. (C) The antennae-enriched candidates (OBPs, CSPs, and SNMPs). FA, female antennae; MA, male antennae; Bo, other body parts. Highlighted histograms: the male-biased ORs (blue); the female-biased ORs (orange); the non-sex-biased ORs (green). Error bars represent standard error. Different small letters on bars indicate significant differences $(p<0.05$, ANOVA, HSD). 
DmelOBP59a found in D. melanogaster (Pikielny et al., 1994; Galindo and Smith, 2001; Wu et al., 2015; Sun et al., 2018). Both OBP83b and Lush have been reported to play roles in sensing semiochemical attractants and the pheromone 11-cis vaccenyl acetate (Xu et al., 2005; Wu et al., 2016). DmelOBP59a has been reported with a function in hygroreception (Sun et al., 2018). HillOBP1, 2, 9, and 11 are expressed only in antennae, similar to what has been observed in other Dipterans. OBP genes present in the same genomic cluster show same pattern of expression in antennae. Two pairs of OBPs HillOBP1/2, HillOBP12/17 are predominantly expressed in antennae and are organized in tandem on scaffold 1521 and 2279, respectively. Based on the phylogeny in Figure 1, it suggest that there has been recent gene duplication. In addition to genes encoding OBPs, a gene encoding a CSP, HillCSP5, is also exclusively expressed in antennae. Therefore, HillOBP1, 2, 11 , and HillCSP5 are likely to play a role in antennal chemicalrecognition.

A total of $70 \mathrm{OR}$ unigenes are identified from $H$. illucens antennae, which is a greater number than those identified from the antennae of other Dipterans, such as B. dorsalis (60 OR genes identified) (Jin et al., 2017) C. stygia (50) (Leitch et al., 2015) Episyrphus balteatus (51) and Eupeodes corollae (42) (Wang et al., 2017) D. melanogaster (39) (Menuz et al., 2014) Scaeva pyrastri (38) (Li et al., 2016), and Chlorops oryzae (25) (Qiu et al., 2018). The high number of ORencoding unigenes may be associated with its ability for diverse host-odor detection in H. illucens. Surprisingly, except Orco, no OR orthologs to the identified $H$. illucens $\mathrm{OR}$ genes are found from other Dipteran species. Apparently, a speciesspecific expansion has happened to form the 69 OR-encoding genes in $H$. illucens (Figure 3). Gene expansion often reflects the adaptation of a species to its ecological niche. This major OR gene expansion seems to indicate that $H$. illucens require multiple OR genes to detect a diversity of structurally similar odorants in its living habitat. In addition, several ORs within tandem arrays including HillOR2/3/7/14/23/40/47/55/60, HillOR24/29/33/41/49, and HillOR13/19/26/31/43, form the same phylogenetic clade, respectively (Figure 3 ). It is likely to result from a relatively recent local gene duplication.

ORs expressed predominantly in female antennae are predicted to function in oviposition-related odorant (Pelletier et al., 2010) or male released pheromones detection (Anderson et al., 2009). ORs expressed equally in the male and female antennae are predicted to function in the detection of general odorants such as feeding attractants (Yan et al., 2015). HillOR32, $44,56,57,60$, and 66 are predominantly expressed in female antennae, and therefore are likely involved in regulation of female-specific behaviors, such as localization of oviposition sites and responses to the pheromones released by males. HillOR2$6,9,10,14,15,19,39,40,52,59$, and 63 are predominantly expressed in male antennae, and therefore, may be associated with the detection of female sex pheromones. The remaining ORs are roughly equally expressed in both female and male antennae, and therefore, may be involved in general odorant detection (Figure 7).
Seventeen antennal IR genes are identified in this study from H. illucens. In Drosophila, IR92a, IR84a/8a, IR76b/IR41a, IR75a/IR8a, IR64a/IR8a have been reported to be involved in, respectively, sensing ammonia and amines (Min et al., 2013) phenylacetaldehyde and phenylacetic acid (Grosjean et al., 2011) polyamines (Hussain et al., 2016) acetic acid (Prieto-Godino et al., 2016), and other acids (Ai et al., 2010). IR93a/IR68a/IR40a and IR21a/IR25a have been reported to be responsible for, respectively, temperature and moisture detection (Knecht et al., 2016, 2017) and cool sensing (Ni et al., 2016). IR genes identified here in $H$. illucens show sequence similarity to some of these characterized IRs and they may have similar functions.

Ten genes encoding GRs are identified from this study in H. illucens, which is similar to the $12 \mathrm{GR}$ genes reported in the antennae of D. melanogaster (Menuz et al., 2014). However, the number of GRs found in $\mathrm{H}$. illucens is much fewer than those reported in other Dipteran species, such as C. stygia (21 GR genes reported) (Leitch et al., 2015). GRs are known to function as taste and contact receptors and are often involved in host-specific pollination behavior (Jauker et al., 2009; Lucie et al., 2013). Some of the identified GRs from $H$. illucens show orthologous relationship with GRs from other insect species. For example, HillGR5 is homologous to DmelGR28b, a saponin receptor in D. melanogaster, and HillGR10 is homologous to DmelGR64a, a sugar receptor. Those GR homologs identified here in H. illucens may play similar roles as reported in other insects.

Two SNMP unigenes are identified in this study. Both SNMPs are conserved compared with other holometabolous insect species. SNMP1 is usually expressed in pheromonesensitive olfactory sensory neurons, and mediates responses to lipid pheromones (Jin et al., 2008; Nichols and Vogt, 2008; Vogt et al., 2009; Gomez-Diaz et al., 2016). In H. illucens, SNMP1 is predominantly expressed in antennae, supporting a role of SNMP1 in pheromone detection.

\section{DATA AVAILABILITY STATEMENT}

All data generated during this study are included in this article/Supplementary Material.

\section{AUTHOR CONTRIBUTIONS}

QX performed the experiments. ZW analyzed the data. QX, ZW, $\mathrm{XA}$, and $\mathrm{XZ}$ wrote and revised the manuscript.

\section{FUNDING}

This project was supported by GDAS Special Project of Science and Technology Development (2019GDASYL-0302007), Science and Technology Planning Project of Guangdong Province (2017B030314047), Guangdong Key Laboratory of Animal Conservation and Resource Utilization (GIABR-KF201701), and Science and Technology Program of Guangzhou (201508020108 and 201805010008). 


\section{ACKNOWLEDGMENTS}

We are grateful to Dr. Mingshun Chen (Kansas State University, United States) for editorial assistance and comments on the manuscript.

\section{SUPPLEMENTARY MATERIAL}

The Supplementary Material for this article can be found online at: https://www.frontiersin.org/articles/10.3389/fphys. 2020.00720/full\#supplementary-material

FIGURE S1 | Transcriptome overview of $H$. illucens antennae. (A) Unigenes annotated in NCBI-nr, NCBI-nt, Swiss-Prot, GO, COG and KEGG databases. (B) Species distribution of the top $H$. illucens unigenes best BLASTx hits. BLAST analysis against the non-redundant protein database was performed with a cut-of $E$-value of $10^{-5}$. (C) Gene ontology (GO) term assignment of the $H$. illucens unigenes.

FIGURE S2 | Multiple amino acid alignment of HillOBPs.

FIGURE S3 | Multiple amino alignment of HillCSPS.

FIGURE S4 | Maximum likelihood of candidate SNMPs from H. illucens and other Dipteran insects. Branch support was estimated using 1000 bootstrap replicates,

\section{REFERENCES}

Ai, M., Blais, S., Park, J. Y., Min, S., Neubert, T. A., and Suh, G. S. (2013). Ionotropic glutamate receptors IR64a and IR8a form a functional odorant receptor complex in vivo in Drosophila. J. Neurosci. 33, 10741-10749. doi: 10.1523/jneurosci.5419-12.2013

Ai, M., Min, S., Grosjean, Y., Leblanc, C., Bell, R., Benton, R., et al. (2010). Acid sensing by the Drosophila olfactory system. Nature 468:691. doi: 10.1038/ nature 09537

Andersen, C. L., Jensen, J. L., and Ørntoft, T. F. (2004). Normalization of realtime quantitative reverse transcription-PCR data: a model-based variance estimation approach to identify genes suited for normalization, applied to bladder and colon cancer data sets. Cancer Res. 64, 5245-5250. doi: 10.1158/0008-5472.can04-0496

Anderson, A. R., Wanner, K. W., Trowell, S. C., Warr, C. G., Jaquin-Joly, E., Zagatti, P., et al. (2009). Molecular basis of female-specific odorant responses in Bombyx mori. Insect Biochem. Mol. Biol. 39, 189-197. doi: 10.1016/j.ibmb.2008.11.002

Andersson, M. N., Videvall, E., Walden, K. K., Harris, M. O., Robertson, H. M., and Lofstedt, C. (2014). Sex- and tissue-specific profiles of chemosensory gene expression in a herbivorous gall-inducing fly (Diptera: Cecidomyiidae). BMC Genomics 15:501. doi: 10.1186/1471-2164-15-501

Benton, R., Vannice, K. S., Gomez-Diaz, C., and Vosshall, L. B. (2009). Variant ionotropic glutamate receptors as chemosensory receptors in Drosophila. Cell 136, 149-162. doi: 10.1016/j.cell.2008.12.001

Benton, R., Vannice, K. S., and Vosshall, L. B. (2007). An essential role for a CD36-related receptor in pheromone detection in Drosophila. Nature 450:289. doi: $10.1038 /$ nature 06328

Booth, D. C., and Sheppard, C. (1984). Oviposition of the black soldier fly, Hermetia illucens (Diptera: Stratiomyidae): eggs, masses, timing, and site characteristics. Environ. Entomol. 13, 421-423. doi: 10.1093/ee/13.2.421

Brammer, C. A., and von Dohlen, C. D. (2007). Evolutionary history of stratiomyidae (Insecta: Diptera): the molecular phylogeny of a diverse family of flies. Mol. Phylogenet. Evol. 43, 660-673. doi: 10.1016/j.ympev.2006.09.006

Bray, S., and Amrein, H. (2003). A putative Drosophila pheromone receptor expressed in male-specific taste neurons is required for efficient courtship. Neuron 39, 1019-1029. doi: 10.1016/s0896-6273(03)00542-7

Chen, C., Buhl, E., Xu, M., Croset, V., Rees, J. S., Lilley, K. S., et al. (2015). Drosophila ionotropic receptor 25 a mediates circadian clock resetting by temperature. Nature 527, 516-520. doi: 10.1038/nature 16148 and bootstrap values were displayed with color circles at the branch nodes. The scale bar indicate the expected number of amino acid substitutions per site.

\section{TABLE S1 | Chemosensory genes from Dipteran species.}

TABLE S2 | Candidate chemosensory genes and reference gene of $H$. illucens antennal transcriptome. Sheet 1: Unigenes of candidate OBP with gene name, sequence information, best BLASTx hit in NCBI-nr database, predicted domains and FPKM, etc. Sheet 2: Unigenes of candidate CSPs. Sheet 3: Unigenes of candidate ORs. Sheet 4: Unigenes of candidate IRs. Sheet 5: Unigenes of candidate GRs. Sheet 6: Unigenes of candidate SNMPs. Sheet 7: Unigenes of reference genes.

TABLE S3 | Amino acid sequences of $H$. illucens and other insect used in the phylogenetic analyses.

TABLE S4 | Ranking of the six reference genes of $H$. illucens using Normfinder and BestKeeper in different tissues.

TABLE S5 | Primer pairs used for RT-PCR and RT-qPCR.

TABLE S6 | Summary of the transcriptome sequencing and assembly process.

TABLE S7 | Distribution of unigene length interval in the $H$. illucens antennal transcriptome assembly.

TABLE S8 | A list of the expression level of all unigenes in H. illucens antennae. Expression level is listed in order from highest to lowest.

Clyne, P. J., Warr, C. G., and Carlson, J. R. (2000). Candidate taste receptors in Drosophila. Science 287, 1830-1834. doi: 10.1126/science.287.5459.1830

Clyne, P. J., Warr, C. G., Freeman, M. R., Lessing, D., Kim, J., and Carlson, J. R. (1999). A novel family of divergent seven-transmembrane proteins: candidate odorant receptors in Drosophila. Neuron 22, 327-338. doi: 10.1016/s08966273(00)81093-4

Cui, Y., Kang, C., Wu, Z., and Lin, J. (2019). Identification and expression analyses of olfactory gene families in the rice grasshopper, oxya chinensis, from antennal transcriptomes. Front. Physiol. 10:1223. doi: 10.3389/fphys.2019.01223

Dahanukar, A., Lei, Y., Kwon, J. Y., and Carlson, J. R. (2007). Two Gr genes underlie sugar reception in Drosophila. Neuron 56, 503-516. doi: 10.1016/j.neuron.2007. 10.024

Galindo, K., and Smith, D. P. (2001). A large family of divergent Drosophila odorant-binding proteins expressed in gustatory and olfactory sensilla. Genetics 159, 1059-1072

Gomez-Diaz, C., Bargeton, B., Abuin, L., Bukar, N., Reina, J. H., Bartoi, T., et al. (2016). A CD36 ectodomain mediates insect pheromone detection via a putative tunnelling mechanism. Nat. Commun. 7:11866.

Gomez-Diaz, C., Reina, J. H., Cambillau, C., and Benton, R. (2013). Ligands for pheromone-sensing neurons are not conformationally activated odorant binding proteins. PLoS Biol. 11:e1001546. doi: 10.1371/journal.pbio.1001546

Gorter, J. A., Jagadeesh, S., Gahr, C., Boonekamp, J. J., Levine, J. D., and Billeter, J. C. (2016). The nutritional and hedonic value of food modulate sexual receptivity in Drosophila melanogaster females. Sci. Rep. 6:19441.

Grabherr, M. G., Haas, B. J., Yassour, M., Levin, J. Z., Thompson, D. A., Amit, I., et al. (2011). Full-length transcriptome assembly from RNA-Seq data without a reference genome. Nat. Biotechnol. 29, 644-652. doi: 10.1038/nbt.1883

Grosjean, Y., Rytz, R., Farine, J. P., Abuin, L., Cortot, J., Jefferis, G. S., et al. (2011). An olfactory receptor for food-derived odours promotes male courtship in Drosophila. Nature 478, 236-240. doi: 10.1038/nature10428

Hekmat-Scafe, D. S., Scafe, C. R., McKinney, A. J., and Tanouye, M. A. (2002). Genome-wide analysis of the odorant-binding protein gene family in Drosophila melanogaster. Genome Res. 12, 1357-1369. doi: 10.1101/gr.23 9402

Hussain, A., Zhang, M., Ucpunar, H. K., Svensson, T., Quillery, E., Gompel, N., et al. (2016). Ionotropic chemosensory receptors mediate the taste and smell of polyamines. PLoS Biol. 14:e1002454. doi: 10.1371/journal.pbio.1002454

Jauker, F., Diekötter, T., Schwarzbach, F., and Wolters, V. (2009). Pollinator dispersal in an agricultural matrix: opposing responses of wild bees and 
hoverflies to landscape structure and distance from main habitat. Landscape Ecol. 24, 547-555. doi: 10.1007/s10980-009-9331-2

Jiao, Y., Moon, S. J., and Montell, C. (2007). A Drosophila gustatory receptor required for the responses to sucrose, glucose, and maltose identified by mRNA tagging. Proc. Natl. Acad. Sci. U.S.A. 104, 14110-14115. doi: 10.1073/pnas. 0702421104

Jin, S., Zhou, X., Gu, F., Zhong, G., and Yi, X. (2017). Olfactory plasticity: variation in the expression of chemosensory receptors in Bactrocera dorsalis in different physiological states. Front. Physiol. 8:672. doi: 10.3389/fphys.2019.0672

Jin, X., Ha, T. S., and Smith, D. P. (2008). SNMP is a signaling component required for pheromone sensitivity in Drosophila. Proc. Natl. Acad. Sci. U.S.A. 105, 10996-11001. doi: 10.1073/pnas.0803309105

Jones, W. D., Cayirlioglu, P., Kadow, I. G., and Vosshall, L. B. (2007). Two chemosensory receptors together mediate carbon dioxide detection in Drosophila. Nature 445, 86-90. doi: 10.1038/nature05466

Kain, P., Boyle, S. M., Tharadra, S. K., Guda, T., Christine, P., Dahanukar, A., et al. (2013). Odour receptors and neurons for DEET and new insect repellents. Nature 502, 507-512. doi: 10.1038/nature12594

Katoh, K., and Standley, D. M. (2013). MAFFT multiple sequence alignment software version 7: improvements in performance and usability. Mol. Biol. Evol. 30, 772-780. doi: $10.1093 / \mathrm{molbev} / \mathrm{mst} 010$

Kearse, M., Moir, R., Wilson, A., Stones-Havas, S., Cheung, M., Sturrock, S., et al. (2012). Geneious Basic: an integrated and extendable desktop software platform for the organization and analysis of sequence data. Bioinformatics 28 , 1647-1649. doi: 10.1093/bioinformatics/bts199

Knecht, Z. A., Silbering, A. F., Cruz, J., Yang, L., Croset, V., Benton, R., et al. (2017). Ionotropic Receptor-dependent moist and dry cells control hygrosensation in Drosophila. eLife 6:26654.

Knecht, Z. A., Silbering, A. F., Ni, L., Klein, M., Budelli, G., Bell, R., et al. (2016) Distinct combinations of variant ionotropic glutamate receptors mediate thermosensation and hygrosensation in Drosophila. eLife 5:e17879.

Koh, T. W., He, Z., Gorur-Shandilya, S., Menuz, K., Larter, N. K., Stewart, S., et al. (2014). The Drosophila IR20a clade of ionotropic receptors are candidate taste and pheromone receptors. Neuron 83, 850-865. doi: 10.1016/j.neuron.2014. 07.012

Kwon, J. Y., Dahanukar, A., Weiss, L. A., and Carlson, J. R. (2007). The molecular basis of $\mathrm{CO} 2$ reception in Drosophila. Proc. Natl. Acad. Sci. U.S.A. 104, 35743578. doi: 10.1073/pnas.0700079104

Leal, W. S. (2013). Odorant reception in insects: roles of receptors, binding proteins, and degrading enzymes. Annu. Rev. Entomol. 58, 373-391. doi: 10. 1146/annurev-ento-120811-153635

Leitch, O., Papanicolaou, A., Lennard, C., Kirkbride, K. P., and Anderson, A. (2015). Chemosensory genes identified in the antennal transcriptome of the blowfly Calliphora stygia. BMC Genomics 16:255. doi: 10.1186/1471-216415-255

Li, X. M., Zhu, X. Y., He, P., Xu, L., Sun, L., Chen, L., et al. (2016). Molecular characterization and sex distribution of chemosensory receptor gene family based on transcriptome analysis of Scaeva pyrastri. PLoS One 11:e155323. doi: 10.1371/journal.pbio. 155323

Livak, K. J., and Schmittgen, T. D. (2001). Analysis of relative gene expression data using real-time quantitative PCR and the 2-DELTADELTACT method. Methods 25, 402-408. doi: 10.1006/meth.2001.1262

Lucie, R., Manuel, P., and Aude, V. (2013). Migration and dispersal may drive to high genetic variation and significant genetic mixing: the case of two agriculturally important, continental hoverflies (Episyrphus balteatus and Sphaerophoria scripta). Mol. Ecol. 22, 5329-5339. doi: 10.1111/mec.12483

Macharia, R., Mireji, P., Murungi, E., Murilla, G., Christoffels, A., Aksoy, S., et al. (2016). Genome-Wide comparative analysis of chemosensory gene families in five tsetse fly species. PLoS Negl. Trop. Dis. 10:e4421. doi: 10.1371/journal.pbio. 04421

Menuz, K., Larter, N. K., Park, J., and Carlson, J. R. (2014). An RNA-seq screen of the Drosophila antenna identifies a transporter necessary for ammonia detection. PLoS Genet. 10:e1004810. doi: 10.1371/journal.pbio.1004810

Min, S., Ai, M., Shin, S. A., and Suh, G. S. (2013). Dedicated olfactory neurons mediating attraction behavior to ammonia and amines in Drosophila. Proc. Natl. Acad. Sci. U.S.A. 110, E1321-E1329.

Newton, G. L., Sheppard, D. C., Watson, D. W., Burtle, G. J., Dove, C. R., Tomberlin, J. K., et al. (2005). "The black soldier fly, Hermetia illucens, as a manure management/resource recovery tool," in Proceedings of the Symposium on the State Of The Science Of Animal Manure And Waste Management, New York, NY.

Ni, L., Klein, M., Svec, K. V., Budelli, G., Chang, E. C., Ferrer, A. J., et al. (2016). The ionotropic receptors IR21a and IR25a mediate cool sensing in Drosophila. eLife 5:13254.

Nichols, Z., and Vogt, R. G. (2008). The SNMP/CD36 gene family in Diptera, Hymenoptera and Coleoptera: Drosophila melanogaster, D-pseudoobscura, Anopheles gambiae, Aedes aegypti, Apis mellifera, and Tribolium castaneum. Insect Biochem. Mol. Biol. 38, 398-415.

Pelletier, J., Hughes, D. T., Luetje, C. W., and Leal, W. S. (2010). An odorant receptor from the southern house mosquito Culex pipiens quinquefasciatus sensitive to oviposition attractants. PLoS One 5:e10090. doi: 10.1371/journal. pbio. 10090

Pfaffl, M. W., Tichopad, A., Prgomet, C., and Neuvians, T. P. (2004). Determination of stable housekeeping genes, differentially regulated target genes and sample integrity: bestkeeper - Excel-based tool using pair-wise correlations. Biotechnol. Lett. 26, 509-515. doi: 10.1023/b:bile.0000019559.84305.47

Pikielny, C. W., Hasan, G., Rouyer, F., and Rosbash, M. (1994). Members of a family of Drosophila putative odorant-binding proteins are expressed in different subsets of olfactory hairs. Neuron 12, 35-49. doi: 10.1016/0896-6273(94) 90150-3

Price, M. N., Dehal, P. S., and Arkin, A. P. (2010). FastTree 2-approximately maximum-likelihood trees for large alignments. PLoS One 5:e9490. doi: 10. 1371/journal.pbio.009490

Prieto-Godino, L. L., Rytz, R., Bargeton, B., Abuin, L., Arguello, J. R., Peraro, M. D., et al. (2016). Olfactory receptor pseudo-pseudogenes. Nature 539, 93-97. doi: 10.1038/nature19824

Qiu, L., Tao, S., He, H., Ding, W., and Li, Y. (2018). Transcriptomics reveal the molecular underpinnings of chemosensory proteins in Chlorops oryzae. BMC Genomics 19:890. doi: 10.1186/1471-2164-15-890

Quevillon, E., Silventoinen, V., Pillai, S., Harte, N., Mulder, N., Apweiler, R., et al. (2005). InterProScan: protein domains identifier. Nucleic Acids Res. 33, W116-W120

Rinker, D. C., Zhou, X., Pitts, R. J., Rokas, A., and Zwiebel, L. J. (2013). Antennal transcriptome profiles of anopheline mosquitoes reveal human host olfactory specialization in Anopheles gambiae. BMC Genomics 14:749. doi: 10.1186/ 1471-2164-15-749

Robertson, H. M., Warr, C. G., and Carlson, J. R. (2003). Molecular evolution of the insect chemoreceptor gene superfamily in Drosophila melanogaster. Proc. Natl. Acad. Sci. U.S.A. 100, 14537-14542. doi: 10.1073/pnas.2335847100

Sandler, B. H., Nikonova, L., Leal, W. S., and Clardy, J. (2000). Sexual attraction in the silkworm moth: structure of the pheromone-binding-protein-bombykol complex. Chem. Biol. 7, 143-151. doi: 10.1016/s1074-5521(00)00078-8

Sang, J., Rimal, S., and Lee, Y. (2019). Gustatory receptor 28b is necessary for avoiding saponin in Drosophila melanogaster. EMBO Rep. 20:e47328.

Scott, J. G., Warren, W. C., Beukeboom, L. W., Bopp, D., Clark, A. G., Giers, S. D., et al. (2014). Genome of the house fly, Musca domestica L., a global vector of diseases with adaptations to a septic environment. Genome Biol. $15: 466$.

Sievers, F., Wilm, A., Dineen, D., Gibson, T. J., Karplus, K., Li, W., et al. (2011). Fast, scalable generation of high-quality protein multiple sequence alignments using clustal omega. Mol. Syst. Biol. 7:539. doi: 10.1038/msb.2011.75

Silbering, A. F., Rytz, R., Grosjean, Y., Abuin, L., Ramdya, P., Jefferis, G. S., et al. (2011). Complementary function and integrated wiring of the evolutionarily distinct Drosophila olfactory subsystems. J. Neurosci. 31, 13357-13375. doi: 10.1523/jneurosci.2360-11.2011

Stewart, S., Koh, T. W., Ghosh, A. C., and Carlson, J. R. (2015). Candidate ionotropic taste receptors in the Drosophila larva. Proc. Natl. Acad. Sci. U.S.A. 112, 4195-4201. doi: 10.1073/pnas.1503292112

$\mathrm{Su}$, C., and Carlson, J. R. (2013). Neuroscience. Circuit logic of avoidance and attraction. Science 340, 1295-1297. doi: 10.1126/science.1240139

Sun, J. S., Larter, N. K., Chahda, J. S., Rioux, D., Gumaste, A., and Carlson, J. R. (2018). Humidity response depends on the small soluble protein Obp59a in Drosophila. eLife 7:e39249.

Sung, H. Y., Jeong, Y. T., Lim, J. Y., Kim, H., Oh, S. M., Hwang, S. W., et al. (2017). Heterogeneity in the Drosophila gustatory receptor complexes that detect aversive compounds. Nat. Commun. 8:1484. 
Tauxe, G. M., MacWilliam, D., Boyle, S. M., Guda, T., and Ray, A. (2013). Targeting a dual detector of skin and CO2 to modify mosquito host seeking. Cell 155, 1365-1379. doi: 10.1016/j.cell.2013.11.013

Tomberlin, J. K., and Sheppard, D. C. (2001). Lekking behavior of the black soldier fly (Diptera: Stratiomyidae). Fla. Entomol. 84, 729-730.

Tomberlin, J. K., and Sheppard, D. C. (2002). Factors influencing mating and oviposition of black soldier flies (Diptera: stratiomyidae) in a colony. J. Entomol. Sci. 37, 345-352. doi: 10.18474/0749-8004-37.4.345

Trapnell, C., Williams, B. A., Pertea, G., Mortazavi, A., Kwan, G., van Baren, M. J., et al. (2010). Transcript assembly and quantification by RNA-Seq reveals unannotated transcripts and isoform switching during cell differentiation. Nat. Biotechnol. 28, 511-515. doi: 10.1038/nbt.1621

Turner, S. L., and Ray, A. (2009). Modification of CO2 avoidance behaviour in Drosophila by inhibitory odorants. Nature 461, 159-277.

Vieira, F. G., and Rozas, J. (2011). Comparative genomics of the odorant-binding and chemosensory protein gene families across the arthropoda: origin and evolutionary history of the chemosensory system. Genome Biol. Evol. 3, 476490. doi: 10.1093/gbe/evr033

Vogt, R. G., Miller, N. E., Litvack, R., Fandino, R. A., Sparks, J., Staples, J., et al. (2009). The insect SNMP gene family. Insect Biochem. Mol. Biol. 39, 448-456. doi: 10.1016/j.ibmb.2009.03.007

Vosshall, L. B., Amrein, H., Morozov, P. S., Rzhetsky, A., and Axel, R. (1999). A spatial map of olfactory receptor expression in the Drosophila antenna. Cell 96, 725-736. doi: 10.1016/s0092-8674(00)80582-6

Wang, B., Liu, Y., and Wang, G. R. (2017). Chemosensory genes in the antennal transcriptome of two syrphid species, Episyrphus balteatus and Eupeodes corollae (Diptera: Syrphidae). BMC Genomics 18:586. doi: 10.1186/1471-216415-586

Wojtasek, H., and Leal, W. S. (1999). Degradation of an alkaloid pheromone from the pale-brown chafer, Phyllopertha diversa (Coleoptera: Scarabaeidae), by an insect olfactory cytochrome P450. FEBS Lett. 458, 333-336. doi: 10.1016/s00145793(99)01178-3
Wu, Z., Lin, J., Zhang, H., and Zeng, X. (2016). BdorOBP83a-2 mediates responses of the oriental fruit fly to semiochemicals. Front. Physiol. 7:452. doi: 10.3389/ fphys.2019.0452

Wu, Z., Zhang, H., Wang, Z., Bin, S., He, H., and Lin, J. (2015). Discovery of chemosensory genes in the oriental fruit fly, Bactrocera dorsalis. PLoS One 10:e129794. doi: 10.1371/journal.pbio.129794

Xu, P. X., Atkinson, R., Jones, D., and Smith, D. P. (2005). Drosophila OBP LUSH is required for activity of pheromone-sensitive neurons. Neuron 45, 193-200. doi: 10.1016/j.neuron.2004.12.031

Yan, S. W., Zhang, J., Liu, Y., Li, G. Q., and Wang, G. R. (2015). An olfactory receptor from Apolygus lucorum (Meyer-Dur) mainly tuned to volatiles from flowering host plants. J. Insect Physiol. 79, 36-41. doi: 10.1016/j.jinsphys.2015. 06.002

Zhan, S., Fang, G., Cai, M., Kou, Z., Xu, J., Cao, Y., et al. (2020). Genomic landscape and genetic manipulation of the black soldier fly Hermetia illucens, a natural waste recycler. Cell Res. 30, 50-60. doi: 10.1038/s41422-0190252-6

Zheng, L., Crippen, T. L., Holmes, L., Singh, B., Pimsler, M. L., Benbow, M. E., et al. (2013). Bacteria mediate oviposition by the black soldier fly, Hermetia illucens (L.), (Diptera: Stratiomyidae). Sci. Rep. $3: 2563$.

Conflict of Interest: The authors declare that the research was conducted in the absence of any commercial or financial relationships that could be construed as a potential conflict of interest.

Copyright (c) $2020 \mathrm{Xu}, \mathrm{Wu}$, Zeng and An. This is an open-access article distributed under the terms of the Creative Commons Attribution License (CC BY). The use, distribution or reproduction in other forums is permitted, provided the original author(s) and the copyright owner(s) are credited and that the original publication in this journal is cited, in accordance with accepted academic practice. No use, distribution or reproduction is permitted which does not comply with these terms. 